\title{
Security Based Network for Health Care System
}

\author{
Jae Yoon Lee1), Lahari Kolasani2)
}

\begin{abstract}
In this project we chiefly finished the support of mobile cloud computing, wireless body area networks will be considerably increased for large readying of pervasive care applications. However, several technical problems and challenges square measure associated with the combination of WBANs and MCC. In this article, we study a cloud-enabled WBAN design and its applications in pervasive care systems. We highlight the methodologies for transmittal important sign information to the cloud by victimisation energy-efficient routing, cloud resource allocation, semantic interactions, and data security mechanisms. Pervasive healthcare applications utilizing body device networks generate a large quantity of information that require to be managed and hold on for process and future usage. Cloud computing concept is a new trend for economical managing and process of device information on-line. This paper presents a platform based on Cloud Computing for management of mobile and wearable care sensors, demonstrating this way the cloud paradigm applied on pervasive care.
\end{abstract}

Keywords : sensors, WBAN, semantic, interactions, MCC.

\section{Introduction}

The introduction of the pervasive attention paradigm has enabled the attention towards the freelance living of senior people and additionally the would love for constant medical direction of chronic patients or habitants at remote, isolated or underserved locations[1-3]. during this context, advanced electronic attention services area unit required to be created on the market through a network anytime, anywhere and to anyone[4-6]. A medical assistive setting on the different hand considerations the use of pervasive and omnipresent technologies for delivering the on top of services[7]. Wireless technologies enable the real time transmission of knowledge a couple of patient's condition to caregivers[8-11]. Numerous moveable devices square measure on the market that will notice sure medical conditions-pulse rate, blood pressure, breath alcohol level, and so on-from a user's bit. However this development and preparation of

Received(January 06, 2015), Review Result(1st: January 20, 2011, 2nd: February 17, 2011), Accepted(March 10, 2015)

${ }^{1}$ Department of Applied Computer Engineering, Dankook University, 152 Jukjeon-ro, Suji-gu, Gyeonggi-do, Korea email: ultrastory@gmail.com

${ }^{2}$ (Corresponding Author) Vidyaa Vikas Educational Institutions, Mysore - Bannur Road, Alanahally, Mysuru, Karnataka, India email: hilahari@gmail.com 
health data management through mobile devices introduces many challenges: knowledge storage and management (e.g., physical storage issues, availability and maintenance), interoperability and convenience of heterogeneous resources, security and privacy (e.g., permission control, data namelessness, etc.), unified and ubiquitous access square measure a few to say One potential resolution for addressing all said problems is that the introduction of Cloud Computing construct in electronic tending systems. Cloud Computing provides the facility to access shared resources and customary infrastructure in an exceedingly ubiquitous and clear method, offering services on-demand, over the network, and performing operations that meet dynamical desires.

Due to several recent technological advances and new ideas, such as wireless body area networks (WBANs) and low-power wireless communications, pervasive health monitoring and management services square measure turning into more and more standard. However, efficient management of the massive variety of monitored knowledge collected from numerous WBANs is a very important issue for his or her large-scale adoption in pervasive tending services. Since WBANs have limited memory, energy, computation, and communication capabilities, they require a powerful and ascendable superior computing and large storage infrastructure for real-time operation and knowledge storage, as well as for online and offline knowledge analysis. Mobile cloud computing (MCC) is gradually turning into a promising technology, which provides a versatile stack of large computing, storage, and software services in a ascendable and virtualized manner at low value. The integration of WBANs and MCC is predicted to facilitate the event of cost-effective, scalable, and data-driven pervasive healthcare systems, which should be in a position to notice long health observation and knowledge analysis of patients in several environments.

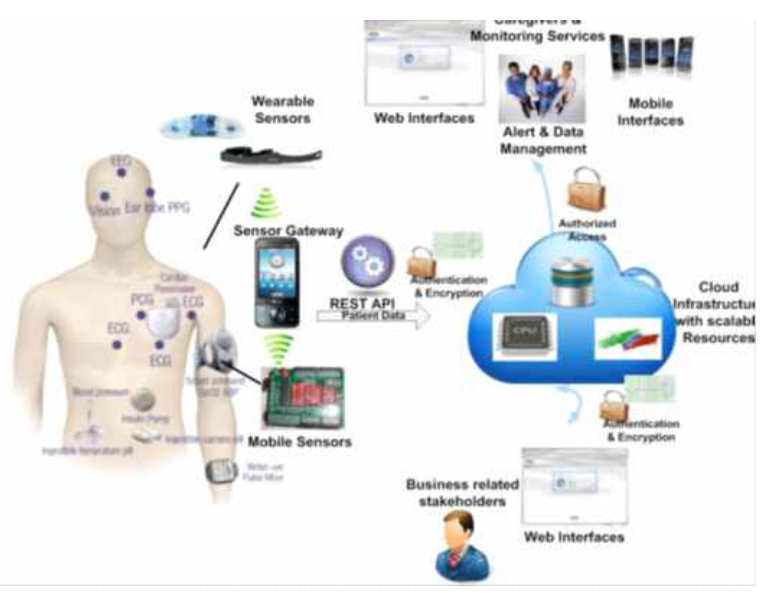

[Fig. 1] Mobile Cloud Computing 


\section{MCC benefits}

MCC inherits many advantages of cloud computing such as dynamic provisioning, scalability, and ease of integration, as well as of a mobile network, such as seamless mobility[6]. We will develop and deploy varied mobile applications for pervasive tending, which will access larger and quicker information storage services and process power from the cloud. Furthermore, MCC can improve the dependableness and security of mobile applications, where the information and computation will be protected within the medical cloud.

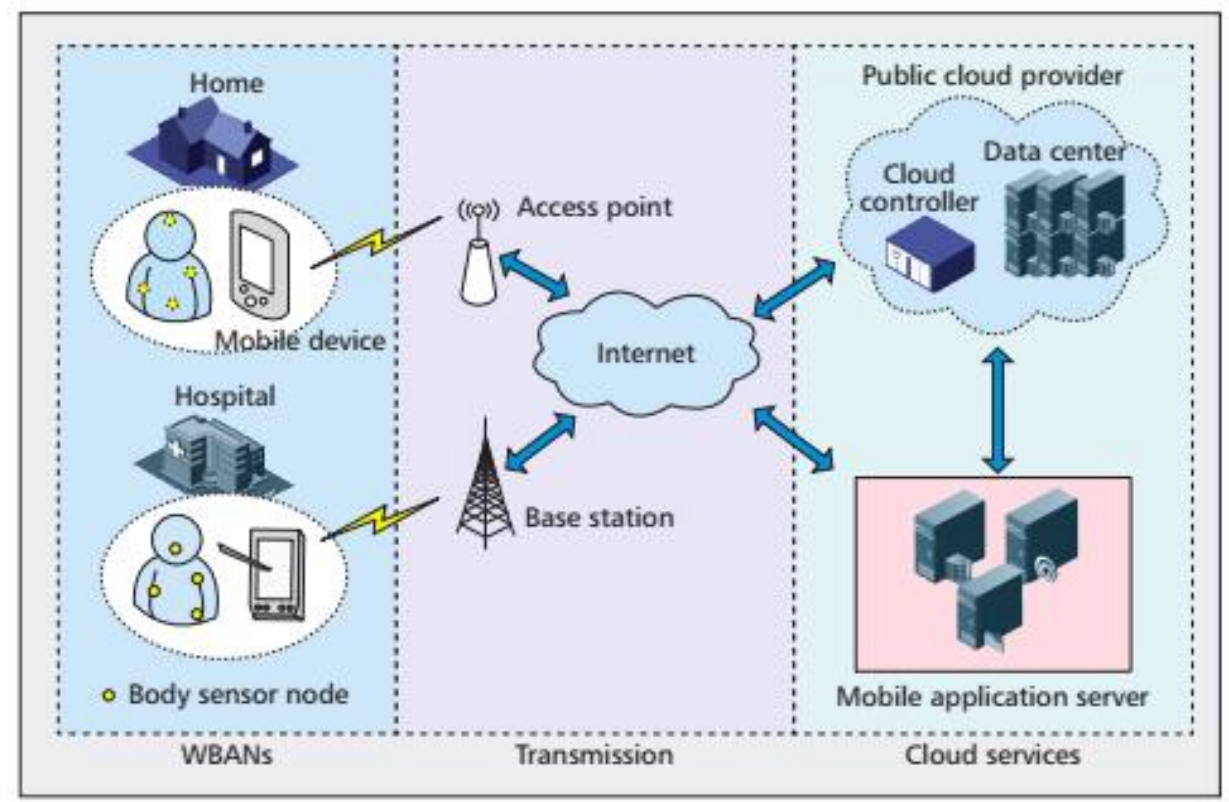

[Fig. 2] Conceptual architecture for WBANs with MCC capability

Many mobile applications together with mobile commerce, mobile learning, and mobile gaming have been developed for various MCC environments. As an example, a prototype of a mobile tending info management system based mostly on cloud computing associated a mobile terminal running an humanoid OS is being enforced. This prototype platform is developing services that utilize the Amazon S3 cloud storage service to manage patient health records and medical pictures. 


\section{Proposed System}

In order to overcome all the disadvantages of the algorithm is blowfish algorithm. It can store large amount of data and security is provide by using the 32 bit key. Blowfish is a symmetric encryption algorithm, meaning that it uses the same secret key to both encrypt and decrypt messages. Blowfish is also a block cipher, meaning that it divides a message up into fixed length blocks during encryption and decryption. The block length for Blowfish is 64 bits. Blowfish requires about $5 \mathrm{~KB}$ of memory. A careful implementation on a 32-bit processor can encrypt or decrypt a 64-bit message in approximately 12 clock cycles. Longer messages increase computation time in a linear fashion.

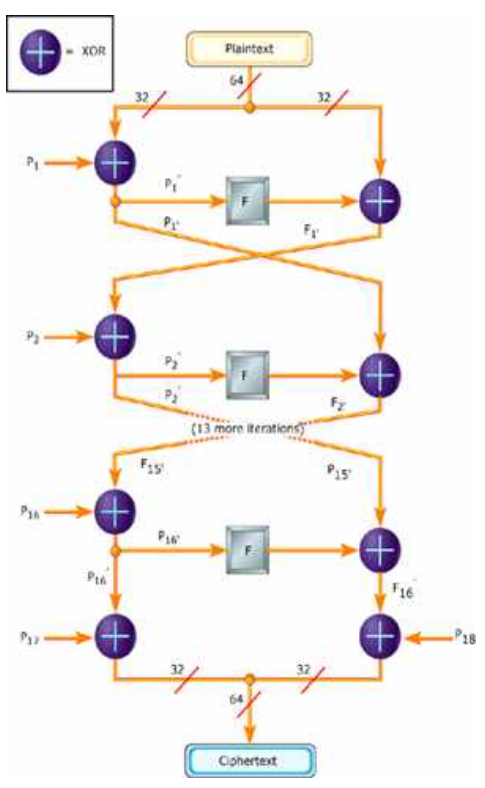

[Fig. 3] Blowfish algorithm 1

In this description, a 64-bit plaintext message is initial divided into thirty two bits. The "left" thirty two bits area unit XOR dysfunction with the primary part of a P-array to form a price $\mathrm{i}$ will decision $\mathrm{P}^{\prime}$, run through a metamorphosis perform known as $\mathrm{F}$, then $\mathrm{XOR}$ dysfunction with the "right" thirty two bits of the message to provide a replacement worth i will decision $\mathrm{F}^{\prime} . \mathrm{F}^{\prime}$ then replaces the "left" half the message and $\mathrm{P}^{\prime}$ replaces the "right" ["fr1], and also the method is perennial fifteen additional times with serial members of the P-array. 
The resulting $\mathrm{P}^{\prime}$ and $\mathrm{F}^{\prime}$ are then XOR ed with the last two entries in the P-array (entries 17 and 18), and recombined to produce the 64-bit cipher text.

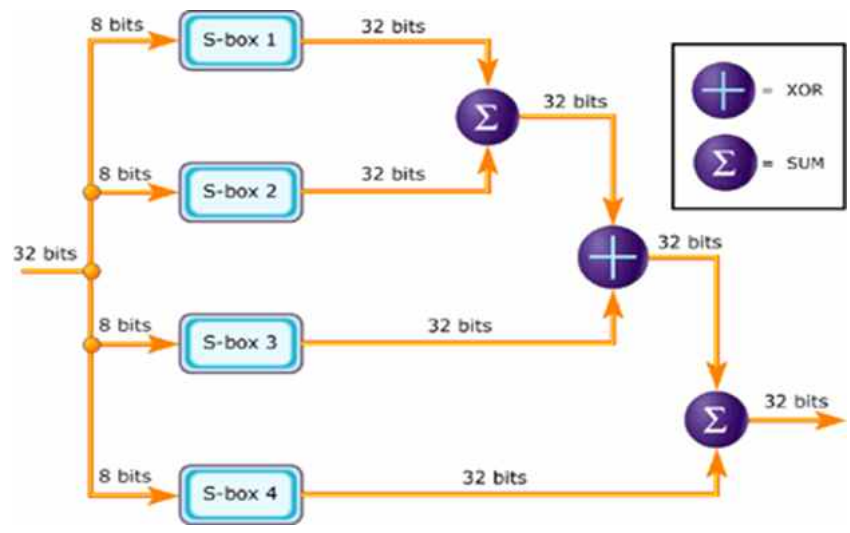

[Fig. 4] Blowfish algorithm 2

The P-array and S-array values used by Blowfish are precomputed based on the user's key. In effect, the user's key is transformed into the P-array and S-array; the key itself may be discarded after the transformation. The P-array and S-array need not be recomputed.

\section{Conclusion}

Pervasive healthcare applications generate a Brobdingnagian quantity of device information that require to be managed properly for more analysis and process. Cloud computing through its elasticity and facility to access shared resources and common infrastructure in an exceedingly present and pervasive manner may be a promising resolution for economical management of pervasive health care information. The presented system is distinctive as an obsessive resolution for managing patient-related information on the cloud which utilizes each open hardware and open computer code resources for developing the hardware and computer code elements of the platform. The seamless integration of WBANs and MCC provides tremendous opportunities for pervasive healthcare systems. In this article, we offer a quick review and outlook of this promising field, and discuss a cloud-enabled WBAN architecture for pervasive health care systems. In particular, we study the practicality and responsibleness of MCC services. We additionally recommend some future analysis directions to improve performance and QoS of cloud-enabled WBANs. We believe cloud-enabled WBANs can attract 
monumental attention and analysis effort within the close to future.

\section{References}

[1] U. Varshney, Pervasive Healthcare, IEEE Computer Magazine, (2003), Vol.36, No.12, pp.138-140.

[2] Maglogiannis I., Doukas C., Kormentzas G., Pliakas T., Wavelet- Based Compression With ROI Coding Support for Mobile Access to DICOM Images Over Heterogeneous Radio Networks, IEEE Transactions on Information Technology in Biomedicine, (2009), Vol.13, No.4, pp.458-466.

[3] L. Coyle, S. Neely, G. Stevenson, M. Sullivan, S. Dobson and P. Nixon. Sensor fusion-based middleware for smart homes, International Journal of Assistive Robotics and Mechatronics, (2007), Vol.8, No.2, pp.53-60.

[4] N. Streitz and P. Nixon. The Disappearing Computer, The Communication of ACM, (2005), Vol.48, No.3, pp.33-35.

[5] K. Farkas, J. Heidemann and L. Iftode. Intelligent Transportation and Pervasive Computing, IEEE Pervasive Computing, (2006), pp.18-19.

[6] GoGrid Storage Services, http://www.gogrid.com, July 12 (2014).

[7] Schneier on Security, https://www.schneier.com, July 12 (2014).

[8] Pramod G. Joisha and Prithviraj Banerjee, An algebraic array shape inference system for MATLAB, ACM Transactions on Programming Languages and Systems, (2006), Vol.28, No.5, pp.848-907.

[9] W. M. Ching, Program analysis and code generation in an APL/370 compiler, IBM Journal of Research and Development, (1986), Vol.30, No.6, pp.594-602.

[10] Rajiv Gupta, Optimizing array bound checks using flow analysis, ACM Letters on Programming Languages and Systems, (1993), Vol.2, No.1-4, pp.135-150.

[11] Sakshat Virture Lab, http://iitd.vlab.co.in, July 12 (2014). 